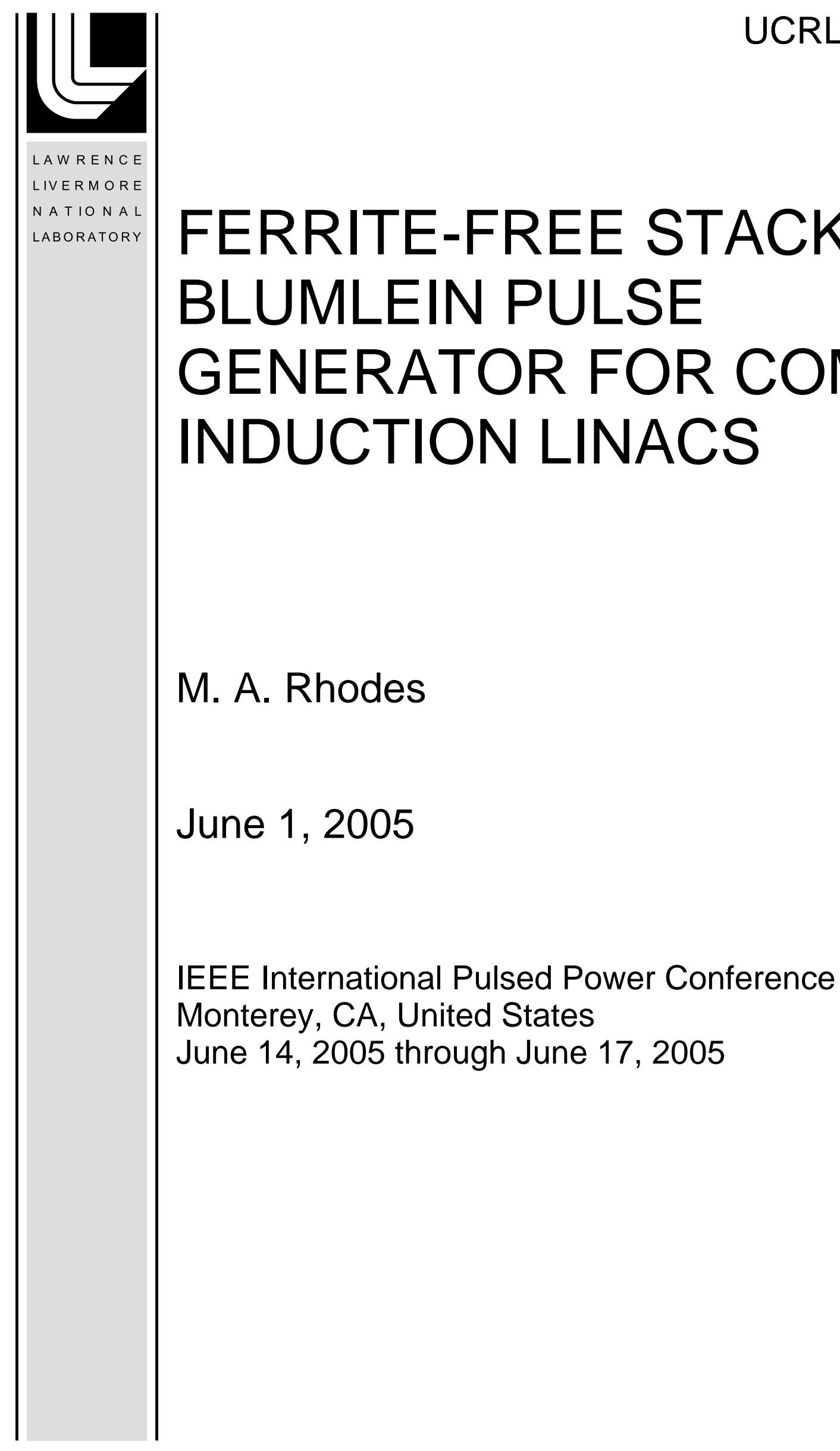


This document was prepared as an account of work sponsored by an agency of the United States Government. Neither the United States Government nor the University of California nor any of their employees, makes any warranty, express or implied, or assumes any legal liability or responsibility for the accuracy, completeness, or usefulness of any information, apparatus, product, or process disclosed, or represents that its use would not infringe privately owned rights. Reference herein to any specific commercial product, process, or service by trade name, trademark, manufacturer, or otherwise, does not necessarily constitute or imply its endorsement, recommendation, or favoring by the United States Government or the University of California. The views and opinions of authors expressed herein do not necessarily state or reflect those of the United States Government or the University of California, and shall not be used for advertising or product endorsement purposes. 


\title{
FERRITE-FREE STACKED BLUMLEIN PULSE GENERATOR FOR COMPACT INDUCTION LINACS*
}

\author{
Mark A. Rhodes \\ Lawrence Livermore National laboratory, PO Box 808, L-485 \\ Livermore, CA, USA
}

\begin{abstract}
Stacked Blumlein Pulse Generators comprised of parallel-plate transmission lines are potentially a useful pulse-power architecture for high-gradient, compact, electron-beam induction accelerators. However, like induction accelerators driven by other pulse-power architectures, it is generally a system requirement that the multi-stage accelerator structure be enclosed in a grounded metal enclosure so that the full beam voltage is not developed on the exterior of the machine. In the past, this has been accomplished by using magnetic cores to prevent the external metal case from shorting the accelerating field. However, magnetic cores are heavy, bulky, expensive, lossy, nonlinear, and therefore generally undesirable.

Various core-free pulse architectures have been reported in the past. One class uses pairs of lines with widely different dielectric constants while another class uses combinations of open-circuit lines combined with short-circuit lines. These designs are encased in metal and support stackable output pulses without the need for magnetic isolation cores. These configurations are also known as bi-polar or zero-integral configurations because they produce a positive and negative voltage pulse with a net time integral of zero. Some of these designs are inefficient leaving substantial stored energy in the lines while others have never been realized as practical accelerating structures.

We present here a particular, realizable, magnetic-corefree induction LINAC geometry that is based on a parallel-plate, stacked Blumlein-like structure, with a symmetric bi-polar, zero integral output pulse, and an outer metal enclosure. Our design is, in theory, $100 \%$ efficient into a matched load. We have evaluated the electromagnetic operation of this geometry by computer modeling. We present the results of this modeling.
\end{abstract}

\section{INTRODUCTION}

We are interested in developing compact linear induction accelerator (LIA) structures for a variety of applications. An LIA is typically constructed in a modular manner with each stage developing an increment of the total accelerator voltage. Since the full voltage for many applications is in the Megavolt range, it is important to keep the outside of the machine at ground potential. This requirement is typically met by using magnetic materials such as ferrite or tape-wound magnetic alloys to increase the inductance of the short-circuit created by the metal case surrounding each accelerator stage. These magnetic materials are never ideal. They are heavy, bulky, expensive, and electrically lossy for the fast, nanosecondrange pulses of interest in many applications. Thus, an LIA accelerator module that is enclosed in metal but does not require a magnetic core to sustain an accelerating voltage would be very advantageous.

Such structures have been proposed in the past [1] [2]. In the literature, such proposed structures are typically presented as generic transmission line diagrams that could be realized in either coaxial or planar geometry. Here we are interested in primarily a design that can be realized in a planar geometry because coaxial realizations are not well suited for stacking multiple stages.

We present here a particular design that is stackable, does not require a magnetic core, and would allow reasonable engineering access to the switches that are always required in real systems.

\section{II.PULSE GENERATING STRUCTURE}

We start with the structure from [1] Fig.2d and shown here as Fig.1. This structure produces a bi-polar pulse of

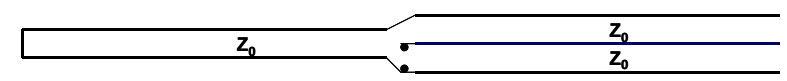

Figure 1. Starting point for the design of a ferrite-free accelerator structure.

$\pm V_{0}$ when charged to $V_{0}$ into a matched load of $2 Z_{0}$ that appears at the second half cycle. It is naturally, fully encased in metal. The shorted-line on the left side is passive in that it is not charged. The switch region, in this structure is not very accessible, so we derive an equivalent structure by splitting the shorted line into two sections and wrapping these shorted sections above and

\footnotetext{
* This work was performed under the auspices of the U.S. Department of Energy by the University of California, Lawrence Livermore National Laboratory under contract No. W-7405-Eng-48
} 
below the active, charged line sections. We show this structure in Fig. 2.

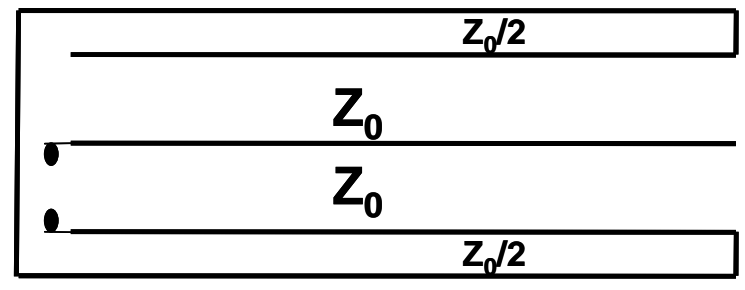

Figure 2. This structure is electrically equivalent to the structure in Fig.1.

By placing the passive, shorted-line sections above and below the charged-line sections, we improve access to the switch-region as the switch is now just inside the metal plate on the left side of the structure. If we realize this structure using planar, parallel-plate transmission lines, we may recognize this structure as a variant of the wellknown stacked Blumlein structure.

To verify the electromagnetic operation of this structure, we use the commercial code XFDTD ${ }^{\text {TM }}$ [3]. This code models structures directly in the time-domain. It allows initial charging of metal plates and allows the use of circuit components such as resistors (for loads) and switches. We constructed a single-stage model of the structure as shown in Fig2.

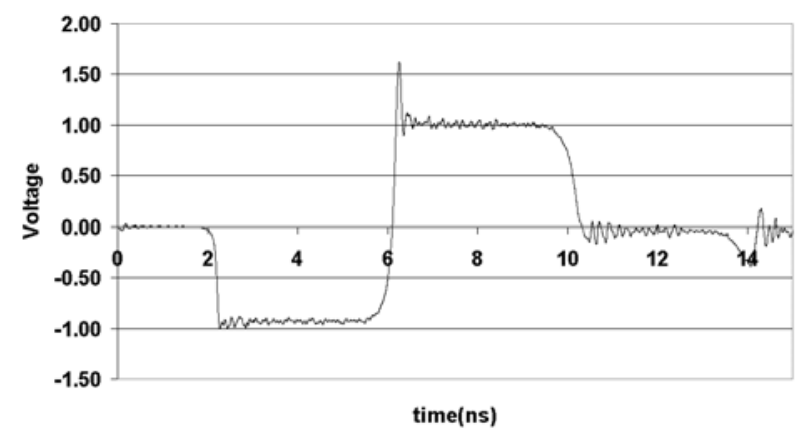

Figure 3. XFDTD output pulse from a model of a single stage.

We set the charged conductor at 1 volt, close the switches at $\mathrm{t}=0$, and close switches in series with the load resistors at $6 \mathrm{~ns}$. The resulting bi-polar voltage pulse is shown n Fig. 3. Notice that although there is a DC short around the outside of the structure, the output pulse is not loaded by the inductance of this path.

\section{ACCELERTOR STRUCTURE}

Using the basic pulse generating structure in the previous section, we now develop a full accelerating structure. We add multiple stages to verify that the voltage adds from each stage. We also improve the azimuthal symmetry placing four identical stacks in a four quadrant cross pattern.

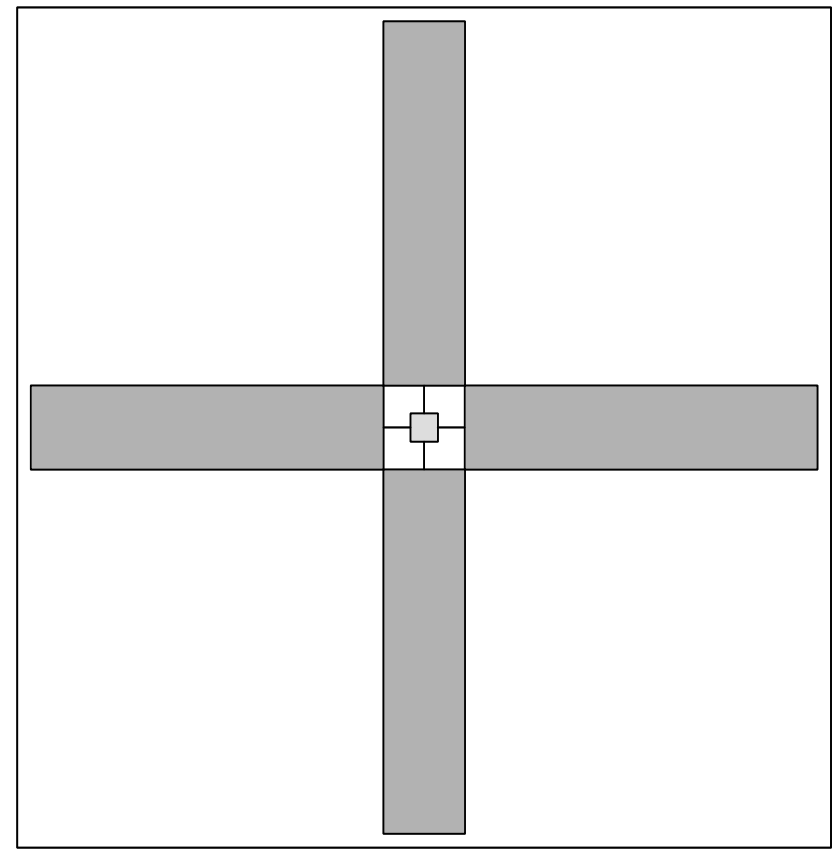

Figure 4. View looking down the bore of the four-stage four-quadrant accelerator structure.

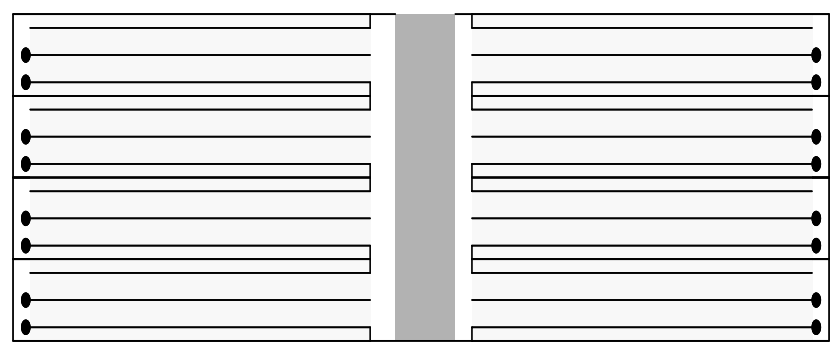

Figure 5. Cross-section view showing the four stages and the central load.

In this model, each quadrant contains a four-stage stack where each stage is of the type described in the previous section. We use an ideal dielectric material with an $\varepsilon_{\mathrm{r}}$ of 10. Each active layer is $40 \mathrm{~cm}$ long, $10 \mathrm{~cm}$ wide, $1 \mathrm{~cm}$ thick, and charged to $10 \mathrm{kV}$. This yields a layer impedance of $11.92 \Omega$ and a one-way transit time of $4.2 n s$. Since each stage has two layers, the stage impedance is $23.84 \Omega$. The passive, shorted lines are $0.5 \mathrm{~cm}$ thick. With four stages in the stack, the impedance of each quadrant is $95.36 \Omega$. Since the four stacks drive the load in parallel, the impedance of the entire structure is $23.84 \Omega$. In the model, we load the structure with a resistive block $4 \mathrm{~cm} \times 4 \mathrm{~cm} \times 12 \mathrm{~cm}$. The conductivity of the block is set at $3.146 \mathrm{~S} / \mathrm{m}$ yielding a net resistance of $23.84 \Omega$ as required. This block is connected to the stack with a metal plate on the bottom and a set of four switches at the top. The switches close after three transit times, which corresponds to the transition time between the positive and negative portions of the voltage pulse. 


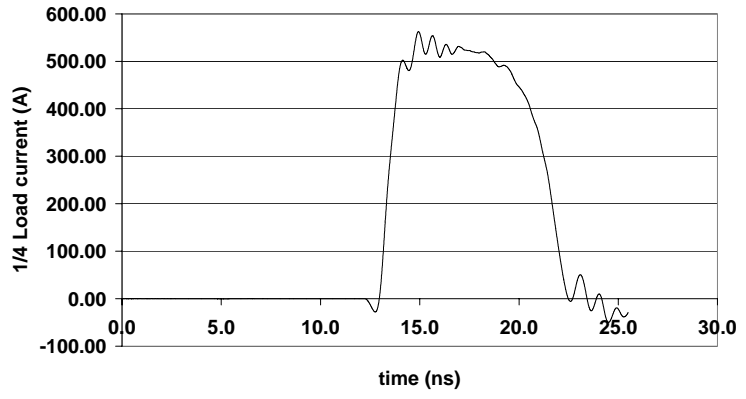

Figure 6. Plot of current in one of four switches connecting resistive load to the stack.

Since the total load current is four times the single quadrant current shown in Fig. 6, the total load current is approximately $2000 \mathrm{~A}$. $47 \mathrm{kV}$ is required to drive this current in a $23.84 \Omega$ load. This a little more than four times the charge voltage in each stage. Therefore, the voltage from each stage adds (more or less) as expected. As with the one-quadrant, single-stage structure, there is a DC short around the outside of the structure yet the pulse is not shorted out by this path.

If we refer again to Fig.4, we can see that at the ends of the structure there is open space in each corner. While the outer metal boundary does DC-short the structure. It does not entirely cover the structure ends. We feel it is possible to build an actual accelerator this way as there is a ground-able boundary on the exterior of the structure. However, for completeness we have created another version of the model where we have added a metallic beam-pipe at each end and completed the end covers.

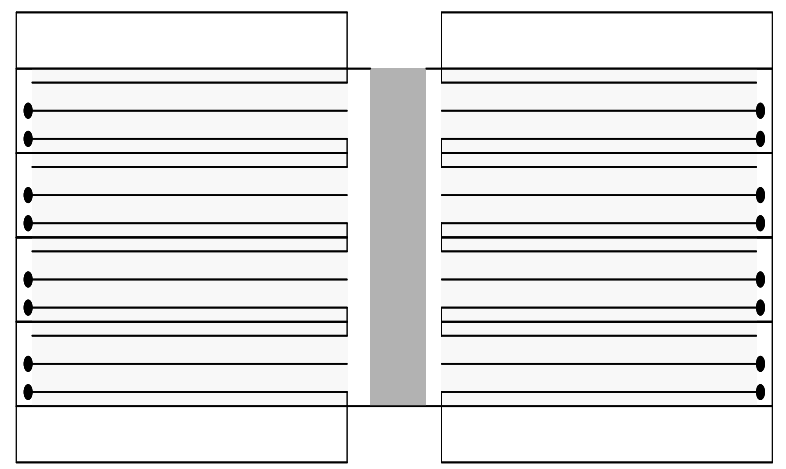

Figure 7. Cross-sectional view where we have added beam-pipes and end covers.

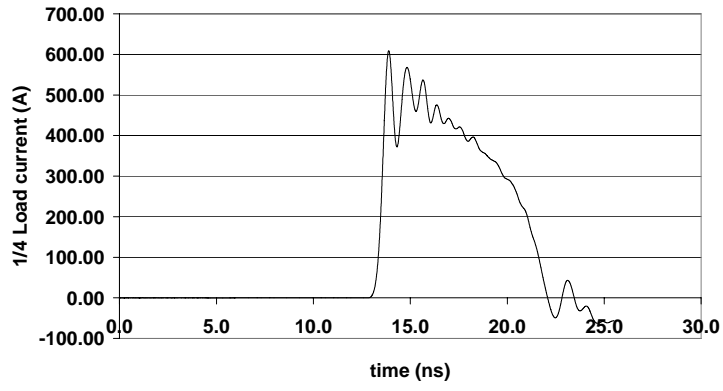

Figure 8. Load current waveform for structure with beampipes and completed end-covers.

We can see in Fig. 8 that complete end-covers load down the output pulse. This is because the end-covers form a transmission line. The voltage developed by the stack drives this transmission line. The net effect appears like inductive loading.

\section{CONCLUSION}

We have presented a variant of a stacked Blumlein-like structure comprised of planar transmission lines that produces a bipolar voltage pulse into a matched load that appears during the second half of the voltage cycle. This structure is surrounded by a ground-able metal boundary yet does not require a magnetic core to keep the metal boundary from shorting the output. We have shown that individual stages may be stacked to achieve higher voltage levels. We have used a commercially available, time-domain, finite-difference code to examine the electromagnetic performance of this structure.

\section{ACKNOWLEDGMENTS}

We would like to acknowledge Scott Nelson for his help in learning how to run the XFDTD code.

\section{REFERENCES}

[1] I. Smith, "Linear Induction Accelerators Made from Pulse-Line Cavities with External Pulse Injection,” Rev. Sci. Instr., vol. 50(6), pp. 714-718, 1979.

[2] M. Ozawa, M. Watanabe, E. Hotta, A. Okino, K. Ko, "Bi-directional Line Pulser System for Linear Induction Accelerators," 12th IEEE International Pulsed Power Conference, vol. 2, pp. 951-954 June 1999.

[3] XFDTD is a product of REMCOM, State College, PA. 